\title{
The role of prior knowledge and curiosity in learning
}

\author{
Shirlene Wade ${ }^{1,2} \cdot$ Celeste Kidd $^{1}$
}

Published online: 11 May 2019

(C) The Psychonomic Society, Inc. 2019

\begin{abstract}
Recent work has argued that curiosity can improve learning. However, these studies also leave open the possibility that being on the verge of knowing can itself induce curiosity. We investigate how prior knowledge relates to curiosity and subsequent learning using a trivia question task. Curiosity in our task is best predicted by a learner's estimate of their current knowledge, more so than an objective measure of what they actually know. Learning is best predicted by both curiosity and an objective measure of knowledge. These results suggest that while curiosity is correlated with knowledge, there is only a small boost in learning from being curious. The implication is that the mechanisms that drive curiosity are not identical to those that drive learning outcomes.
\end{abstract}

Keywords Curiosity $\cdot$ Memory $\cdot$ Learning $\cdot$ Metacognition

\section{Introduction}

Curiosity is considered a strong motivator of learning. While scientific interest in curiosity has persisted for decades (e.g., Berlyne, 1950, 1960; Gruber, Gelman, \& Ranganath, 2014; Henderson \& Moore, 1980; Hutt, 1970; Loewenstein, 1994; Kang et al., 2009), only recently has empirical work shown that curiosity is associated with better learning outcomes (e.g., Gruber et al., 2014; Kang et al., 2009; Reio \& Wiswell, 2000; Stahl \& Feigenson, 2015). Min Jeong Kang et al. (2009) found that the answers to high curiosity-inducing trivia questions were more likely to be recalled than for low curiosity-inducing trivia questions 2 weeks later. Additionally, curiosity was highest when learners had intermediate confidence about the answer to the question - in other words, maximal uncertainty about whether their guess was wrong or right. Functional magnetic resonance imaging (fMRI) results revealed an association between curiosity and increased activation of memory regions: When a participant had guessed an answer incorrectly and the correct answer was revealed, activation in the parahippocampal gyrus and left

Shirlene Wade

sswade91@gmail.com

Celeste Kidd

celestekidd@gmail.com

1 Department of Psychology, University of California, Berkeley, 2121 Berkeley Way, Berkeley, CA 94720-1650, USA

2 Department of Brain and Cognitive Sciences, University of Rochester, Rochester, NY, USA inferior frontal gyrus - regions related to long-term memory consolidation - were modulated by the individual's level of curiosity. In a similar study, high-curiosity states facilitated learning of additional, interleaved stimuli (Gruber et al., 2014). Collectively, these results suggest that curiosity may globally enhance learning by heightening activation in memory regions of the brain. While these studies show a connection between curiosity and learning, why curiosity is associated with learning remains unclear. A clear understanding of this relationship is crucial to designing effective curiosity-based learning interventions, a hot topic right now in the education literature (e.g., Engel, 2011; Gordon, Breazeal, \& Engel, 2015). We do not yet understand even the direction of causality in the relationship between curiosity and learning. Learning could appear enhanced because you are most curious about information that you are on the verge of learning. Thus, the apparent boost to learning may in fact be driven by one's prior knowledge or there may be a feedback loop between the two factors rather than curiosity inherently boosting learning alone.

There's no question that partial knowledge induces curiosity. Infants favor partially learned stimuli in preferentiallooking paradigms (e.g., Aslin, 2007; Haith, 1980; Hunter \& Ames, 1988; Roder, Bushnell, \& Sasseville, 2000). Further, young children favor playing with toys for which they have an incomplete understanding (e.g., Bonawitz, van Schijndel, Friel, \& Schulz, 2012; Cook, Goodman, \& Schulz, 2011; Schulz \& Bonawitz, 2007; Sobel et al., 2007). Curiosity is widely theorized to be triggered by the detection of incomplete knowledge (Loewenstein, 1994). This theory is bolstered by empirical results from the education literature that 
demonstrate relationships between domain-level knowledge and learning (e.g., Alexander, Kulikowich, \& Schulze, 1994; Reio, 2004), as well as recent results that report relationships between adult learners' confidence and their curiosity (e.g., Kang et al., 2009).

It is unclear, then, to what degree the apparent boosts to learning attributed to curiosity may in fact be driven by the partial knowledge itself inducing the curious state. It seems likely that there are bi-directional effects. These kinds of feedback loops are present in the kinds of algorithms used to guide learning in developmental robotics systems (e.g., Baranes \& Oudeyer, 2013; Colas, Fournier, Sigaud, Chetouani, \& Oudeyer, 2018; Forestier, Mollard, \& Oudeyer, 2017), but the existence of such feedback loops has not been empirically tested in human learners.

Here, we aim to clarify the dynamic relationships between partial knowledge, curiosity, and learning using a modified version of a trivia question task. We investigate the degree to which partial knowledge is responsible for curiosity and the observed boosts in learning. We asked participants to take their best guess at trivia questions (Gruber et al., 2014), then provide a judgment of how close they believed their guess to be and a rating of their curiosity for the answer. We also had independent raters judge how close each guess was to the answer as a proxy for an objective measurement of conceptual proximity to the correct response. These data allow us to independently assess the degree to which curiosity and learning are driven by metacognitive estimates of informational uncertainty. Participants were then shown the answer, and later tested on their retention of the said answers. We used these data to comprehensively evaluate the factors that drive curiosity and learning, and the dynamics of these factors that result in boosts to learning.

Our results suggest that while learners' curiosity is best predicted by how close they think they are, their actual learning is predicted by their actual prior knowledge, with a small apparent boost from being curious about the topic. The implication is that the mechanisms that drive learners' curiosity are not identical to those that drive learning outcomes. These results also highlight the important role of metacognition in the processes that guide motivation and learning.

\section{Method}

We recruited 114 participants on Amazon Mechanical Turk based on the expected effect size for curiosity on trivia questions (as estimated via Kang et al., 2009 and Gruber et al., 2014) and how many participants we estimated we would need to exclude (e.g., due to inattention) based on previous laboratory experiments on Amazon Mechanical Turk. From this sample, we excluded 16 participants for providing the same curiosity rating on at least $90 \%$ of trials in the learning phase, and 11 for skipping $20 \%$ or more of trials. ${ }^{1}$ We included the resulting 87 participants in the analyses reported below. Participants received a monetary payment at a rate of US $\$ 10.00$ hourly in exchange for their participation, and the task lasted approximately $50 \mathrm{~min}$.

\section{Stimuli}

We selected trivia questions from the stimulus set of Gruber et al. (2014), which was originally created to produce variability in curiosity responses across trivia questions and individuals (see Appendix A.1. for the specific questions and answers). We presented the questions in a randomized order to participants on Amazon Mechanical Turk through psiTurk (McDonnell et al., 2012).

\section{Task}

The task consisted of three phases: a learning phase, a distractor phase, and a testing phase. Participants were not told that they would be tested on the trivia questions until immediately before the testing phase.

In the learning phase, we presented participants with 100 trivia questions (e.g., "For which president was the blueberry jelly bean made?"), one question at a time (see Appendix A.1.). For each question, participants provided:

(1) Their best guess,

(2) Their estimate of how close their guess was to the correct answer on a scale of 1 (not close) to 7 (very close),

(3) Whether they thought their guess was correct (yes or no), and

(4) Their curiosity about the correct answer on a scale of 1 (not curious) to 7 (very curious).

After submitting their response to a trivia question, participants saw the answer to the trivia question for $5 \mathrm{~s}$. Participants then indicated how surprised they were at seeing the answer, on a scale of 1 (not surprised at all) to 7 (very surprised). Participants were given the option to skip any trials that they did not want to answer. When participants chose to skip a trial, they saw the correct answer for $5 \mathrm{~s}$ and waited 5 more seconds before going to the next trial.

In the distractor phase, participants described their impressions of complex artistic images. Responses were collected but were not analyzed for content. The task served as a buffer task between the learning and testing phase.

In the testing phase, trivia questions were presented in the same order as in the training block. Participants guessed the answer to each question. Participants could skip any trials that

\footnotetext{
${ }^{1}$ We note that including these participants (instead of removing them) does not impact any of the findings reported below.
} 
they did not want to answer. When participants skipped a trial, they were taken to the next trial after a 10 -s waiting period.

\section{Similarity ratings}

Three research assistants from the Rochester Baby Lab rated the similarity of each guess response in the learning and testing phase $(n=6,794)$ to the correct answer on a scale of 1 (not close at all) to 7 (very close). The raters were only provided with the trivia question, correct answer, and participant response (they did not see participant-reported curiosity ratings, participantreported similarity ratings, or whether the responses came from the learning or testing phase). The raters had access to external aids (e.g., information on the internet) in order to make similarity judgments. All raters used the full scale of similarity ratings. The mean similarity rating was 2 out of $7(1.9,2.0$, and 2.1 for each rater), with strong agreement within raters $\left(\mathrm{K}_{w}=0.81\right.$, 0.81 , and 0.84 for each two-rater comparison). To calculate the similarity rating for each item, ratings were $z$-scored by rater and averaged across raters for each item.

\section{Accuracy ratings}

The same research assistants who provided similarity ratings also provided accuracy ratings for all unique responses in the learning phase and test phase. Responses were scored as correct, incorrect, or ambiguous by each rater. Raters were allowed to use external aids (e.g., information on the internet) in order to judge whether a response was accurate or not. For analysis, accuracy of the response was decided based on the majority response. For example, responses were judged to be correct if at least two out of three raters scored the response as correct. There was strong agreement between the three raters (Fleiss' kappa $=0.81$ ).

\section{Analyses and results}

Each participant provided guesses in the learning and testing phase for 100 trivia questions, resulting in a total of 8,700 trials collected for analysis. The average accuracy was $18 \%$ for trials in the learning phase $(M d n=0.16$, range $=0.04$ $0.52)$ and $69 \%$ for trials in the testing phase $(M d n=0.70$, range $=0.27-0.95)$. Participants reported an average curiosity rating of 4.6 out of $7(M d n=4.9$, range $=1.2-6.7)$. Trials were excluded from analysis if the participant guessed correctly in the learning phase ( $n=1,598 / 8,700,18 \%$ of trials), was confident they got an initial guess correct in the learning phase but guessed incorrectly ( $n=1,093 / 8,700,13 \%$ of trials), skipped a response ( $n=4$ in learning phase and $n=17$ in the testing phase) or did not provide an appropriate guess (e.g., wrote "I don't know"; $n=15$ in the learning phase and $n=17$ in the testing phase, for a cumulative total of $<1 \%$ of trials). The analyses reported below are completed on the remaining trials, where the participant indicated that they did not know the answer and guessed the answer incorrectly during the learning phase ( $n=5,973 / 8,700,69 \%$ of trials). ${ }^{2}$ In our final dataset, participants reported an average curiosity rating of 4.4 out of 7 $(M d n=4.6$, range $=1.2-6.9)$. At test, participants were accurate on an average of $61 \%$ of trials $(M=0.61, M d n=0.62$, range $=0.22-0.94)^{3}$

\section{Question 1: What influences curiosity?}

First, we asked how curiosity related to learners' sense of how close their guess was to a correct answer. A Pearson's ProductMoment Correlation yielded a small, significant positive correlation between normalized curiosity and average, $z$-scored similarity rating that the participant provided for each initial guess, $r(5971)=0.23, p<0.0001$. Thus, people appear to be more curious about questions when they believe that their initial guess is closer to the correct answer (see Fig. 1). Next, we asked whether people were curious about answers that they were objectively closer to knowing, as judged by independent raters. A Pearson's Product-Moment Correlation yielded a small, significant correlation, $r(5971)=0.09, p<0.0001$ (see Fig. 2); while the value is statistically significant, the variance explained by the relationship was negligible ( $<1 \%$ of variance). Prior knowledge judged by independent raters was significantly correlated to prior knowledge judged by the learners $r(5971)=0.25, p<$ 0.0001 . To determine whether the observed difference in Pearson correlations between curiosity and our two prior knowledge measures was significant, we followed the recommended procedures in Wilcox (2009). First, we generated bootstrapped confidence intervals from 500 samples of 500 data points. We then calculated the correlations between normalized curiosity ratings, $z$-scored similarity ratings from participants, and z-scored similarity ratings from raters. Finally, we calculated the upper and lower bounds of a confidence interval with Zou's method for overlapping correlations. This interval (0.05-0.20) did not contain 0 , which indicates a significant difference in the magnitude of the two correlations. Collectively, these results show that perceptions of prior knowledge, rather than a more objective measure of prior knowledge, predict curiosity.

\section{Question 2: What influences learning?}

Next, we asked whether the same mechanism that underlies curiosity - perceived prior knowledge - also underlies learning. To address this question, we constructed a mixed-effects

\footnotetext{
${ }^{2}$ See Appendix A.2. for a discussion on the relationship between confidence and curiosity.

${ }^{3}$ All data and analyses are accessible at the following GitHub repository: https://github.com/shirlenewade/PriorKnowledgeCuriosity
} 


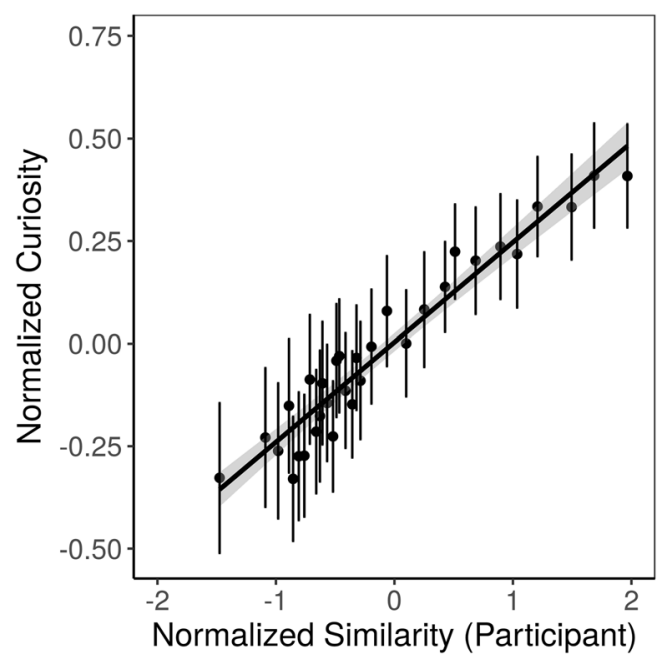

Fig. 1 Curiosity and believed prior knowledge. There was a significant relationship between $z$-scored curiosity ratings ( $y$-axis) and believed prior knowledge ( $x$-axis; $z$-scored participant-provided similarity ratings). Participants were generally more curious about trivia questions when they believed that they were closer to knowing the answer. Each point in the figure represents a binned mean of 181 datapoints (binned $r(31)=0.97, p$ $<0.0001$ ), for visual clarity; however, we note that the main analyses were conducted over the raw data $(r(5971)=0.23, p<0.0001)$. Error bars denote $95 \%$ confidence intervals

logistic regression predicting test accuracy from $z$-scored curiosity ratings and $z$-scored similarity ratings with participant and trial terms included as random effects (see Table 1; estimated model $R^{2}=0.40$ ). Controlling for prior knowledge (estimated by the participant in addition to three independent raters) and

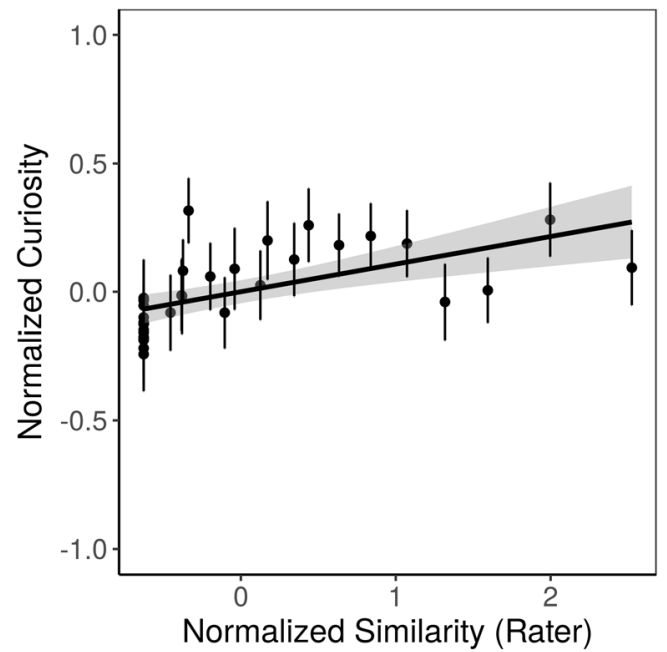

Fig. 2 Curiosity and independent measure of prior knowledge. There was a significant relationship between $z$-scored curiosity ratings ( $y$-axis) and independent judgments of prior knowledge ( $x$-axis; similarity ratings were provided by independent raters, $z$-scored, and averaged). Participants were generally more curious about trivia questions when they were closer to knowing the answer, as judged by three independent raters. Each point on the plot represents a binned mean of 181 data points (binned $r(31)=0.60, p<0.001)$. However, main analyses are performed on the raw data $(r(5971)=0.09, p<0.0001)$. Error bars denote $95 \%$ confidence intervals curiosity, there was a marginal effect of the participant's own estimate of their prior knowledge on learning, $\beta=0.06, S E=$ $0.04, z=1.7, p=0.09$ (see Fig. 3). Participants were marginally more likely to recall a trivia answer at test when they judged their initial guess as closer to the correct answer than when they judged their initial guess as distant from the correct answer. Controlling for the participant's perceived prior knowledge, both curiosity and prior knowledge were significant predictors of test accuracy, $\beta=0.26, S E=0.04, z=7.10, p<0.0001$, and $\beta=0.27$, $S E=0.06, z=4.50, p<0.0001$, respectively. A 1 standard deviation (SD) increase (34.1\%) in $z$-scored curiosity was associated with a $56 \%$ increase in the probability of getting the answer correct (see Fig. 4). Similarly, a 1 SD increase in $z$-scored objective prior knowledge was associated with a 57\% increase in the probability of getting the answer correct (see Fig. 5). This analysis shows that while the learner's perception of prior knowledge predicts the learner's curiosity, it is a marginal predictor of learning outcomes. Rather, we find that both curiosity ratings and a more objective measure of prior knowledge predict learning outcomes.

\section{Results summary}

The results of this experiment help clarify the relationship between curiosity and learning. While previous studies found that test accuracy for trivia questions is linked to a learner's curiosity for the questions (e.g., Gruber et al., 2014; Kang et al., 2009), it was previously unclear whether the learning effects attributed to curiosity would be better explained by curiosity-related variables, such as prior knowledge (e.g., Alexander et al., 1994). We find evidence for both prior knowledge and curiosity providing a boost to learning. Further, we find that curiosity is best predicted by the learner's metacognitive estimate of their own knowledge, while learning is instead better predicted by an objective measure of existing knowledge. In other words, the cognitive mechanisms underlying curiosity and learning are distinct.

Table 1 Regression coefficients from logistic regression predicting learning from curiosity ratings, rater- and participant-provided similarity judgments

\begin{tabular}{lllll}
\hline Term & Coefficient & SE & $Z$ & $p$-value \\
\hline Intercept & 0.93 & 0.19 & 4.86 & $<0.0001 * * *$ \\
Curiosity & 0.26 & 0.04 & 7.10 & $<0.0001 * * *$ \\
Participant similarity & 0.07 & 0.04 & 1.70 & $0.09 *$ \\
Rater similarity & 0.27 & 0.06 & 4.50 & $<0.0001 * * *$ \\
\hline
\end{tabular}

$* p<0.01 * * * p<0.001$ 


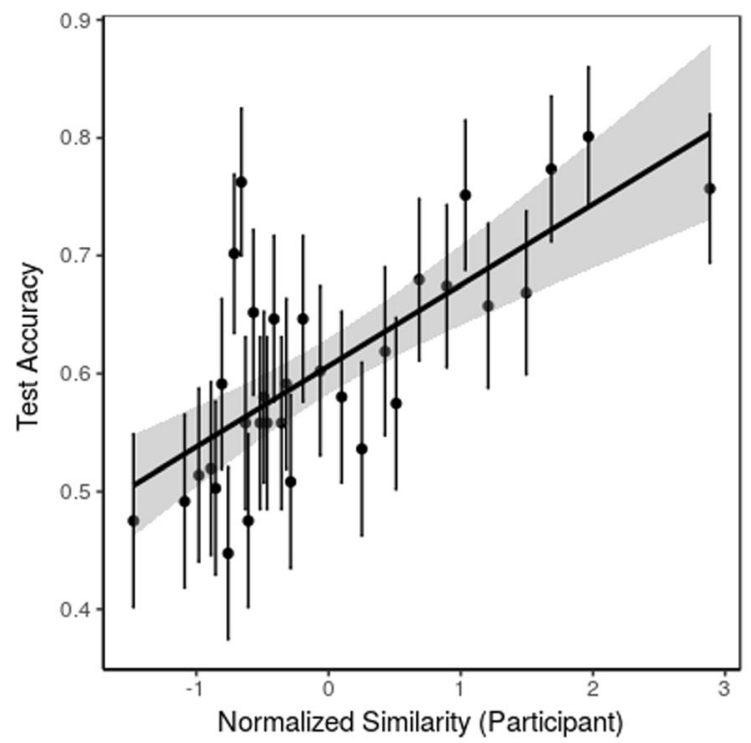

Fig. 3 Participant-estimated prior knowledge marginally predicts test accuracy. Left: A positive linear relationship between perceived prior knowledge ( $x$-axis) and learning ( $y$-axis). Trivia questions were marginally more likely to be recalled correctly at test if participants thought that their initial guess was close, controlling for independent measures of distance-to-learning and initial curiosity. Data points are

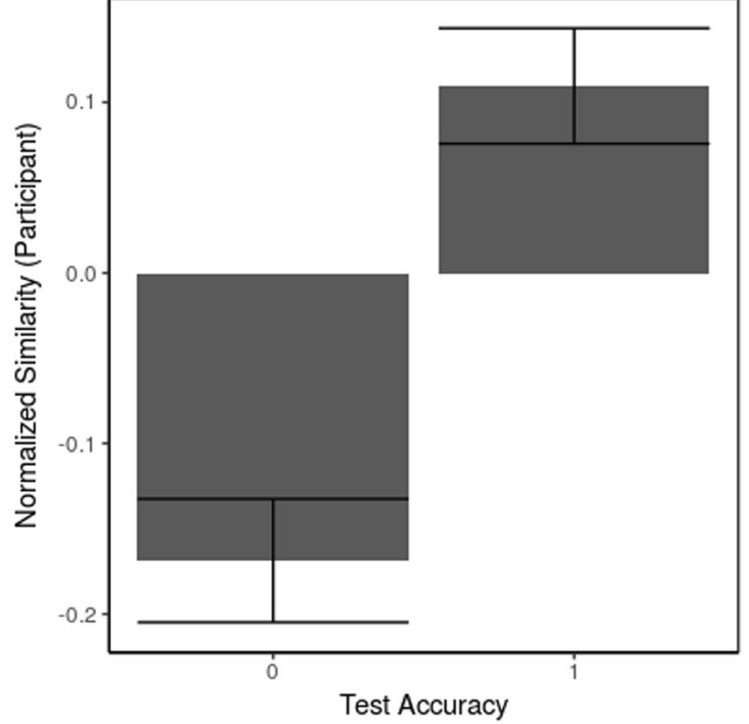

binned means of 181 data points (binned $r(31)=0.72, p<0.001$ ). Right: The average similarity rating participants provided for questions that they recalled correctly (1) or incorrectly (0) at test are shown. In both figures, bars denote $95 \%$ confidence intervals. On average, participants thought they were closer to knowing the answers that they recalled correctly at test compared to answers that they recalled incorrectly at test

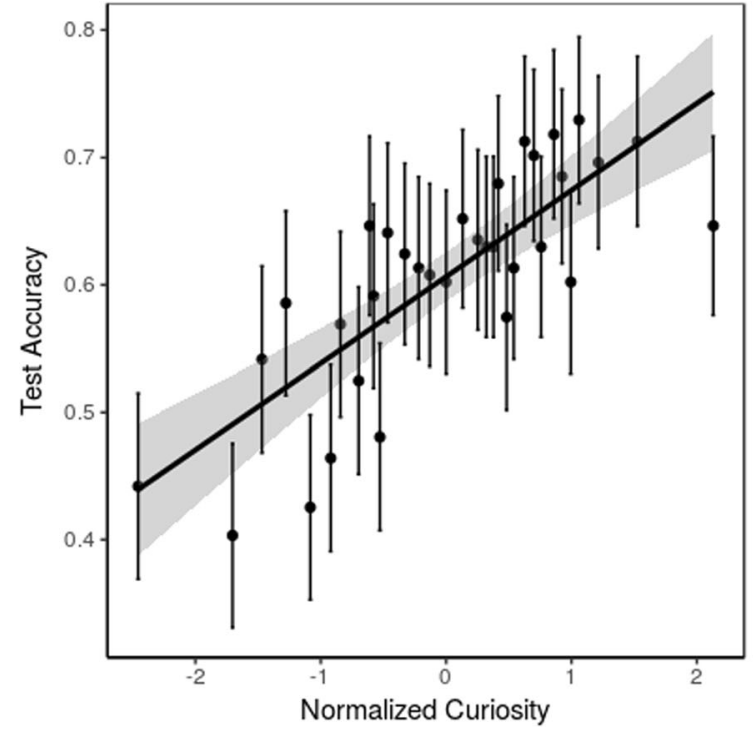

Fig. 4 Learning is associated with greater curiosity. Left: A positive linear relationship between curiosity and test accuracy. Each data point represents the test accuracy ( $y$-axis) and $z$-scored curiosity reported in the learning phase ( $x$-axis) for a single trial. On average, when participants were more curious about a question in the learning phase, they were also more accurate at test. Data points are binned means of 181 data points (binned $r(31)=0.78, p<0.0001)$. Right: The average curiosity rating for

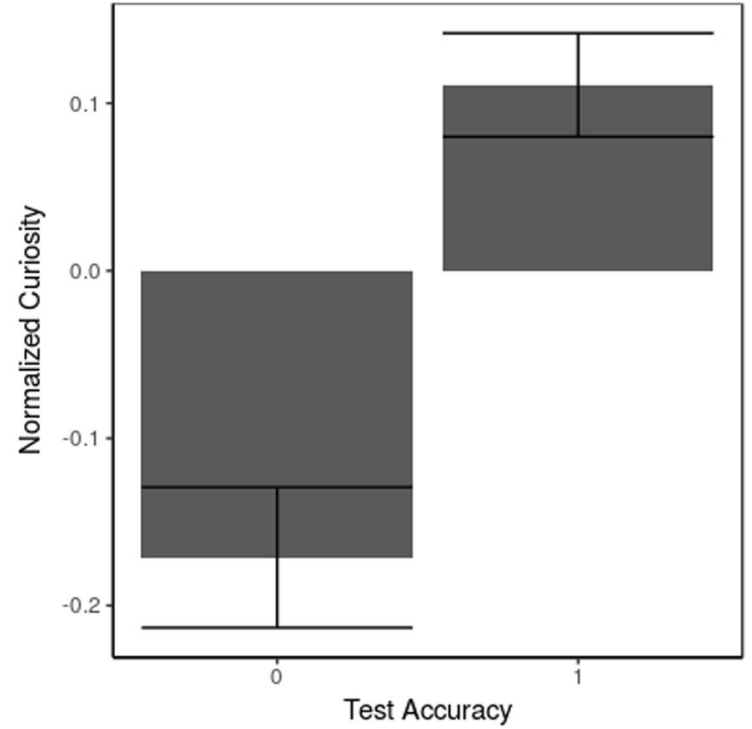

trivia questions that participants recalled correctly (1) and incorrectly (0) at test are shown. In both figures, bars represent $95 \%$ confidence intervals. On average, participants were initially more curious for questions recalled correctly at test compared to those recalled incorrectly. The effect of curiosity on learning was significant when controlling for the effects of prior knowledge and perceived prior knowledge 


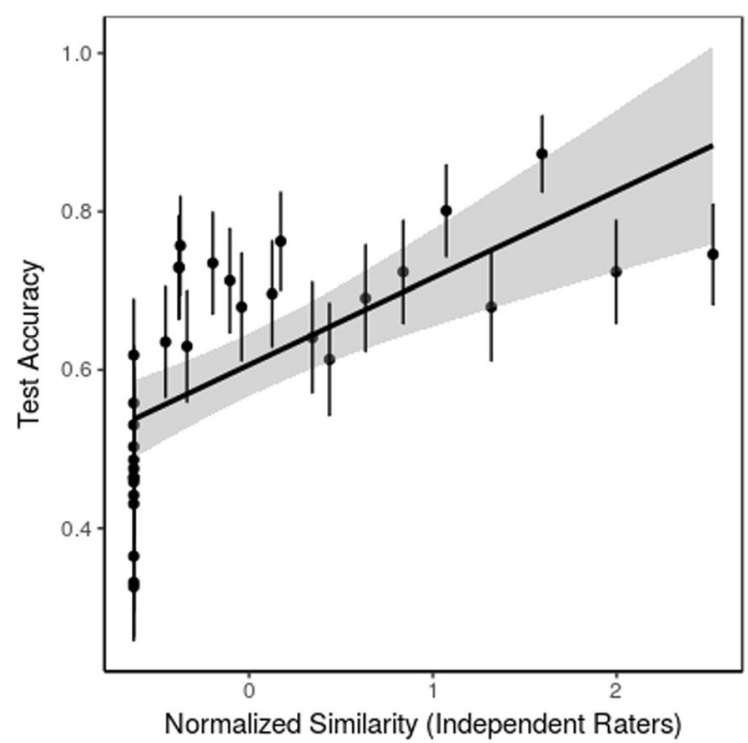

Fig. 5 Rater-estimated prior knowledge predicts test accuracy. Left: A positive relationship between prior knowledge ( $x$-axis) and learning ( $y$ axis). Data points are binned means of 181 data points (binned $r(31)=$ $0.65, p<0.0001)$. Increases in prior knowledge, operationally defined as the similarity of an initial guess to the correct answer as determined by three raters, are associated with better test accuracy. Right: The average similarity rating ( $y$-axis) for trivia answers that were recalled correctly (1)

\section{Discussion}

\section{Curiosity as a metacognitive signal}

Why are learners curious about some things but not others? Our results suggest that learner's metacognitive estimates of their own prior knowledge strongly drive their curiosity. This is an important insight for better understanding of how to motivate learning in an educational context, where previous work has noted that interest, motivation, and background knowledge may each contribute (e.g., Ainley, Hidi, \& Berndorff, 2002; Reio, 2004). Important implications of this work are that learners' curiosity should be piqued when they come to believe that they are close to knowing the answer, and that induced curiosity could boost learning beyond the advantage of just the partial information alone. Our data provide empirical evidence to bolster classic ideas by educators such as Maria Montessori, who argued that educators should aim to provide material that is just a bit beyond the level the learner has already mastered (e.g., the Montessori concept of "readiness", 1964). Our work further builds on these ideas by highlighting the importance of the learner's awareness of where they currently are in the process of knowledge acquisition - the metacognitive dimension of cognitive learning processes. Curiosity may serve as a metacognitive signal that indicates when there's a match between the presented learning material and the learner's readiness to encode it.

It follows that learning outcomes should then depend on individual differences in metacognitive monitoring and

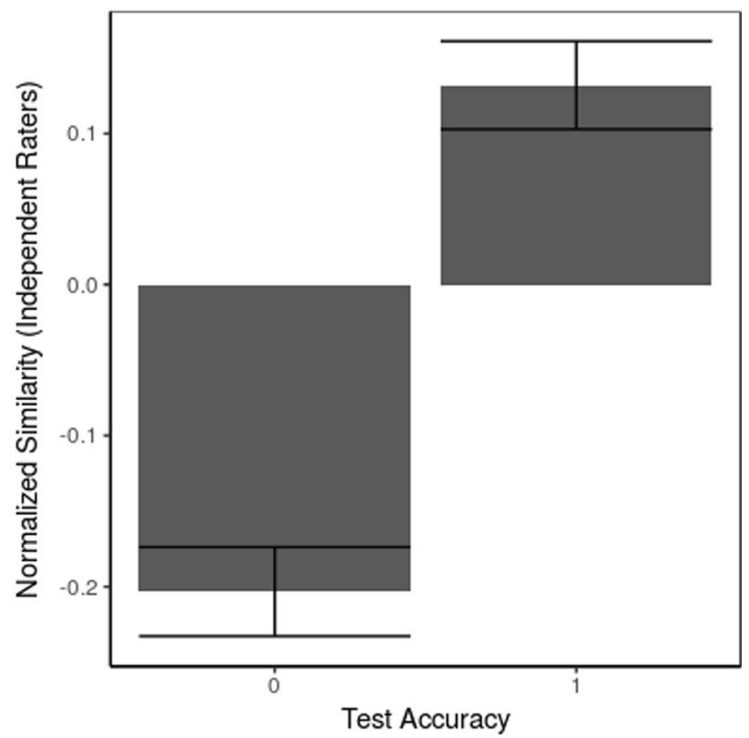

and incorrect (0) at test are shown. Bars denote $95 \%$ confidence intervals. On average, the raters judged initial guesses in the learning phase to be closer to the correct answer when the participant correctly recalled the trivia question at test. The effect of prior knowledge on learning was significant when controlling for the effects of perceived prior knowledge and curiosity

control - the abilities to monitor and act on one's knowledge, respectively. The degree to which these individual differences matter is an important topic for future research, as well as how trait-level differences in curiosity impact learning dynamics.

\section{What exactly are learners curious about?}

These results leave some ambiguity about precisely what type of informational uncertainty is driving the effect. The target of learners' curiosity may primarily be the contents of the perceived information gap, or simply the confirmation of whether or not their best guesses are correct. Our data suggest that learners care about both, and that the learning enhancements apply to the contents regardless. While our primary analyses were conducted only on trials in which individuals believed their guesses to be incorrect, subsequent analyses over all trials (see Appendix Fig. 6) reveal that participants who believed their guess was correct were more curious than those who believed their guess was incorrect. Most likely, both an interest in content and feedback contribute to a learner's overall curiosity. In other words, it's most likely that learners care both about the missing information itself as well as whether or not they guessed right.

\section{Other factors that influence curiosity}

Though the influence of perceived prior knowledge on curiosity is robust, the estimated effect size in our experiment is small, accounting for just $5 \%$ of the variance $(r=0.23)$. Thus, 
other factors may influence individuals' curiosity in the world. Curiosity in real-world circumstances is most likely explained by a combination of multiple high-level factors (e.g., prior knowledge, but also conceptual novelty, topic familiarity, goal relevance) and low-level ones (e.g., visual complexity, salience). We note that curiosity is also influenced by attentional, affect, and mental states. For example, curiosity for science increases after learners watch awe-inducing videos (McPhetres, 2019). Likewise, a reduction in curiosity is one diagnostic criterion for detecting clinical conditions like depression (American Psychiatric Association, 2013) and Alzheimer's (Daffner, Scinto, Weintraub, Guinessey, \& Mesulam, 1992). The interactions between these other factors and prior knowledge is an area ripe for future research.

\section{The feedback loop between curiosity and learning}

In our study, we found a bi-directional relationship between curiosity and learning. First, a learner's perception of their own prior knowledge is related to greater curiosity for new information. Second, curiosity is associated with better learning outcomes. This is particularly interesting given previous work in developmental robotics that suggests that a curiositylearning feedback loop arises out of a single curiosity drive and may maximize learning behaviors and outcomes (e.g., Oudeyer, 2004; Oudeyer \& Kaplan, 2006). Our experiment provides the first empirical result in support of a bi-directional relationship between curiosity and learning in human learners.

Acknowledgements We thank Colin Camerer for helpful discussion; Matthias Gruber for helpful discussion and use of stimuli; Becca Canale, Sarah Field, Rebecca Handsman, Holly Palmeri, and Zoe Pruitt for providing similarity judgements for participant responses; and a Google Faculty Award and the Jacobs Foundation Early Career Research Fellowship to CK for funding in support of this project. This work was also supported by an award from the National Science Foundation Graduate Research Fellowship Program (Grant No. DGE1419118) to SW. Any opinions, findings, and conclusions or recommendations expressed in this material are those of the authors and do not necessarily reflect the views of the National Science Foundation.

\section{Appendix}

\section{Trivia question stimuli}

A subset of 100 trivia questions was selected from Gruber et al.'s (2014) stimuli set

\begin{tabular}{lll}
\hline & Question & Answer \\
1 & $\begin{array}{l}\text { What's the name of the main airport in } \\
\text { Madrid? }\end{array}$ & Barajas \\
2 & $\begin{array}{l}\text { In what city is Texas State University? } \\
3\end{array}$ & San Marcos \\
& What is the state flower of North Dakota? & Wild prairie rose
\end{tabular}

4 What Polish political movement got the support of Pope John Paul II in the 1980s?

5 What leader said in 1942 'Never before have we had so little time in which to do so much'?

$6 \quad$ What song did both Ronald Reagan and Walter Mondale quote in the 1984 presidential campaign?

7 Who was the first democratically elected president of Russia?

8 What was the B-17 long-range bomber nicknamed in World War II?

9 What Pakistani was the first head of state in the 20th century to give birth in office?

10 What Argentinian was buried in a Milan cemetery under the pseudonym Maria Maggi?

11 In which Ohio city was the 1995 Bosnian peace accord signed in?

12 What city's worker-student protests of 1968 resulted in a 33 percent rise in the national minimum wage?

13 Who was the U.S. president when the first edition of the Farmer's Almanac was published?

14 What is the middle item the Cat in the Hat eventually balances in his left hand?

15 What fraternity was George W. Bush in?

Solidarity

Franklin Roosevelt

Born in the USA

Boris Yeltsin

Flying Fortress

Benazir Bhutto

Eva Peron

Dayton

Paris

George

Washington

Red book

Delta Kappa Epsilon

The forint is the monetary unit of what central Hungary European country?

17 What is the name of the largest ocean on Pacific Earth?

18 What's the first word uttered in Citizen Kane? Rosebud

Who hosted the 1994 MTV Movie awards?

Who is the voice of Gary the snail on Spongebob Squarepants?

21 Where was Seth MacFarlane born?

Will Smith

Tom Kenny

Saugus, CA

What color is Mary Jane's shirt during the rain Pink kiss in the first Spider-Man movie?

What is the name of Roy Rogers' horse?

Trigger

What is the name of the goldfish in 'Pinocchio'?

What was actor Lon Chaney also known by?

What cartoon character did Walter Lantz create?

Cleo

Who was the first country artist to sell over 10 million copies of an album?

29 Who appeared on the cover of 'Seventeen' Magazine before selling over 11 million copies of her debut album?

30 Whose guitar version of The Star-Spangled Banner was featured in a 1996 Aiwa TV ad?

What is rocker Darius Rucker's stage name?

What British group got its name from the title of a 1950 Muddy Waters song?

33 What group's lead guitar player is known for his amps with control knobs that go up to 11 ?
The Man of a Thousand Faces

Woody the Woodpecker

Garth Brooks

Whitney Houston

Jimi Hendrix

Hootie

The Rolling Stones

Spinal Tap

God bless America 
What three words preceded 'land that I love' in a 1938 Irving Berlin tune?

35 Which beverage company featured the hit 'Like A Prayer' in its advertisement?

36 Whose 1991 autobiography was called 'And The Beat Goes On'?

37 Which Tour did Prince begin in 1990?

38 Which hit-making band featured Andy Summers and Stewart Copeland?

39 Who collaborated with Michael Bolton on Bolton's 'Steel Bars' hit?

40 What song took over No. 1 from Whitney Houston's 'I Will Always Love You'?

41 Who wrote the song 'I Love Paris'?

Pepsi

Sonny Bono

Nude

The Police

Bob Dylan

A Whole New

World

Cole Porter

42 Whose theme song was 'Where the Blue of the Bing Crosby night Meets the Gold of the Day'?

43 What is the name of the composer who wrote Mozart Don Giovanni?

44 What cartoonist has had three insect species Gary Larson named after him?

45 What is the ninth most poisonous animal in the world?

46 What is the genus of the seahorse?

47 What is the most common cactus?

48 Along with chitin, what strengthens the exoskeleton of bugs?

49 What do birds rely on to swallow?

50 What is 'Turdus migratorius' better known as?

51 What is $10 \%$ of the salt mined in the world used for in America?

52 What radioactive element is extracted from carnotite and pitchblende?

53 What was the first physician to record case histories of patients?

54 Copper and what else are two main constituents of bronze?

55 What colorless gas is essential in the production of fertilizers and light bulbs?

56 What body part is low-density lipoprotein most likely to clog?

57 What are you shopping for if you're sized up by a Brannock Device?

58 What's the unit of capacity for fuel wood? Cord

59 What Internet search utility was named after Gopher the mascot of the University of Minnesota?

60 What heat-resistant element replaced cotton as Tungsten the filament of choice in electric light bulbs in 1910 ?

61 What US coin weighs five grams?

Nickel

62 What chemical compound comes from the Protein Greek word for 'primary'?

63 What type of material constitutes at least half Abrasives of all toothpastes?

64 What is the most commonly known Serotonin tryptamine?

65 What did Jonas Salk invent?

Polio Vaccine

66 Who invented the steamboat Clermont?

67 What was the name of the ship on which Charles Darwin made his scientific voyage?

68 Who invented the radio?
69 Hydrogen and what are thought to be the

Helium primary elements of which Jupiter is composed?

70 What was the name of the first probe to send Viking back pictures from Mars?

71 On which ship did President Nixon welcome Hornet the astronauts back from the Moon?

72 What was the name of the lunar module that Eagle landed the first man on the moon?

73 In horse racing, in which city is the Japan Cup Tokyo held?

74 Yapping Deng was a world champion in which sport?

75 Motor racing's Juan Manuel Fangio came Argentina from which country?

76 Which horse was Horse of the Year from 1960 Kelso to 1964 ?

77 In what game are the standard pieces of Chess Staunton design?

78 What sport uses stones and brooms? Curling

79 What two fruits were Hawaiian women once Coconut and forbidden by law to eat? banana

80 For which president was the blueberry jelly Ronald Reagan bean made?

81 From what were jack-o-lanterns originally Turnips carved?

82 Who was president of the US when Uncle Sam first got a beard?

83 What US president died 79 days after being shot?

84 What nation's 90-man army is the world's oldest, dating back to 1506 ?

85 What president had to read a news ticker to discover that his daughter Luci was engaged?

86 What was the first war in which one jet plane Korean War shot down another?

87 What U.S. president's face graces a $\$ 100,000$ Woodrow Wilson bill?

88 What writer's last words were: 'Last words are Karl Marx for fools who haven't said enough'?

89 What vice president was accused of being Andrew Johnson drunk when sworn in?

90 What inscription on US coins did Theodore In God We Trust Roosevelt try in vain to have removed?

91 Who was stuck in the spacecraft while Neil Michael Collins Armstrong and Buzz Aldrin moon-walked?

92 What is the most shoplifted book? The Bible

93 What state shares its border with the most Tennessee states?

94 What food is the leading source of salmonella Chicken poisoning?

95 Who wrote the Star Spangled Banner? Francis Scott Key

96 What is the second largest island in Europe? Iceland

97 What is Mark Twain's real name? Samuel Clemens

98 Who was first to publish the theory that the Copernicus Earth revolves around the sun?

99 What was the poison used at Socrates' Hemlock execution?

100 Who was the first solo female host of the Academy Awards Ceremony? 


\section{Previous experiment details}

Prior to this experiment, we conducted a similar experiment involving 40 participants recruited on Amazon Mechanical Turk. From this sample, five participants were excluded from analysis due to providing the same curiosity rating for all trials during the learning phase $(n=1)$, internet connectivity issues during the experiment $(n=1)$, and server errors $(n=3)$ that prevented them from completing the task. We presented participants with the same trivia questions used in this experiment. However, there were two differences in the task. First, participants were not asked to indicate how close they thought their guess was to the correct answer. Second, they were asked "Do you know the correct answer?" rather than "Do you think your guess is correct?"

Similar to the current experiment, 3 independent raters provided similarity judgments that were $z$-scored by rater and then averaged for each response. Additionally, we only analyzed trials where the participant indicated that they did not believe that they knew the correct answer.

In both experiments, there was a negligible relationship between curiosity and objective measures of prior knowledge. Curiosity for a trivia answer was influenced by the learner's confidence about their knowledge and the accuracy of their guess. Furthermore, we found independent effects of prior knowledge and curiosity on learning, consistent with the current experiment. However, the relationship between curiosity and confidence depends on how confidence is assessed. In the current experiment, we find that participants are more curious when they believe their initial guess is correct. This is in contrast to the results found in the previous experiment, where

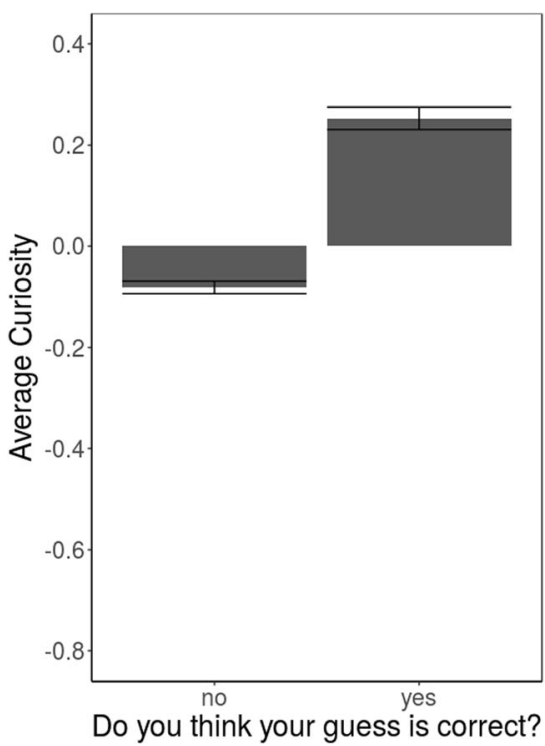

Fig. 6 Curiosity and confidence about knowledge. Bars show the average $z$-scored curiosity ratings ( $y$-axis) for trivia questions as a function of the participant's confidence in their guess ( $x$-axis) in the presented experiment (left plot) and the previous experiment (right plot). The participants were most curious when they believed they did not know the answer (see Appendix Fig. 6).

There are many reasons why the relationship between our curiosity and confidence measures might differ across the two experiments. Importantly, we used two unique questions to assess confidence in the two experiments. For the presented experiment, we asked participants "Do you think that your guess is correct?", while in the previous experiment described in A.2., we asked "Do you know the correct answer?". The relationship between our confidence question and curiosity may differ across experiments because of semantic differences between the two confidence questions. When responding to the question "Do you think that your guess is correct?", participants are specifically judging the accuracy of their response. However, when responding to the question "Do you know the correct answer?", a participant may respond "yes" if they believe that they possess the knowledge, even if they know that their guess is incorrect. In this situation, participants should not respond "yes" to the question "Do you think your guess is correct?". Alternately, the relationship between our confidence question and curiosity may differ due to the change in metacognitive focus in each question. To answer "Do you know the correct answer?", participants may engage in metacognitive monitoring (introspecting about whether they possess knowledge that is currently inaccessible knowledge; Nelson \& Narens, 1990). In contrast, to answer "Do you think your guess is correct?" the participant is asked to focus on a very specific judgment on the accuracy of their guess. This question may have increased participants' desires to guess correctly (see Discussion), leading to greater curiosity when participants believed they were likely to do so (e.g.,

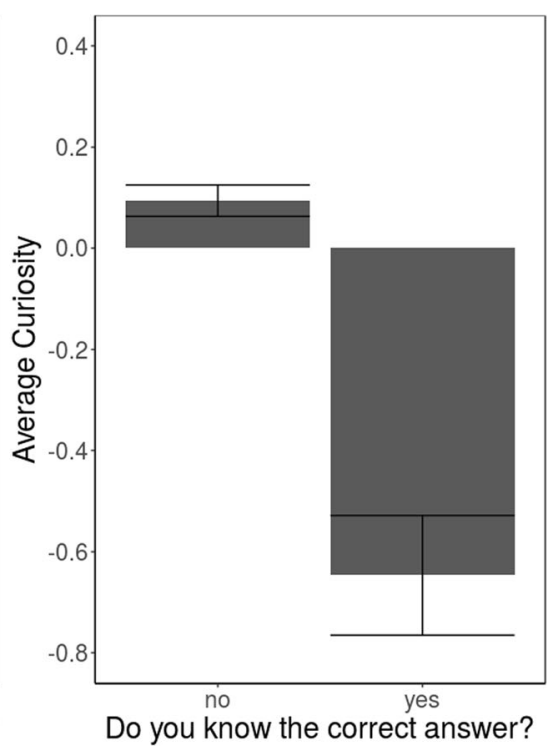

relationship between curiosity and confidence varied depending on the wording of the question ("Do you think your guess is correct?" vs. "Do you know the correct answer?"), suggesting that the questions may have led learners to consider different types of knowledge in their response 
when they indicated that they knew the answer to the question).

Previous work shows that there are many factors that contribute to a learner's judgment of confidence including prior performance on a task (e.g., Marti, Mollica, Piantadosi, \& Kidd, 2019), and behavioral heuristics such as retrieval fluency and ease of processing within the task (e.g., Schwartz \& Metcalfe, 1992; Kornell, 2009, 2014). The relationship between confidence judgments and veridical knowledge varies greatly across domains (e.g., visual domain vs. concept learning; Barthelmé \& Mamassian, 2009; Marti et al., 2019), tasks (e.g., depending on what cues are utilized), and individuals (e.g., Kruger \& Dunning, 1999; Gilbert, 2015). We emphasize that we cannot definitively explain why there is an inverse relationship between curiosity and our confidencemeasuring questions in the two experiments and include these details for others to consider.

\section{References}

Ainley, M., Hidi, S., \& Berndorff, D. (2002). Interest, learning, and the psychological processes that mediate their relationship. Journal of educational psychology, 94(3), 545-561.

Alexander, P. A., Kulikowich, J. M., \& Schulze, S. K. (1994). How subject-matter knowledge affects recall and interest. American Educational Research Journal, 31(2), 313-337.

American Psychiatric Association. (2013). Diagnostic and statistical manual of mental disorders (5th ed.). Arlington, VA: American Psychiatric Publishing.

Aslin, R. N. (2007). What's in a look?. Developmental Science, 10(1), 4853.

Baranes, A., \& Oudeyer, P. Y. (2013). Active learning of inverse models with intrinsically motivated goal exploration in robots. Robotics and Autonomous Systems, 61(1), 49-73.

Barthelmé, S., \& Mamassian, P. (2009). Evaluation of objective uncertainty in the visual system. PLoS Computational Biology, 5(9), e1000504.

Berlyne, D. E. (1950). Novelty and curiosity as determinants of exploratory behaviour. British Journal of Psychology, 41(1-2), 68-80.

Berlyne, D. E. (1960). Conflict, arousal, and curiosity.

Bonawitz, E. B., van Schijndel, T. J., Friel, D., \& Schulz, L. (2012). Children balance theories and evidence in exploration, explanation, and learning. Cognitive Psychology, 64(4), 215-234.

Colas, C., Fournier, P., Sigaud, O., Chetouani, M., \& Oudeyer, P. Y. (2018). CURIOUS: Intrinsically Motivated Multi-Task, MultiGoal Reinforcement Learning. arXiv preprint arXiv:1810.06284.

Cook, C., Goodman, N. D., \& Schulz, L. E. (2011). Where science starts: Spontaneous experiments in preschoolers' exploratory play. Cognition, 120(3), 341-349.

Daffner, K. R., Scinto, L. F. M., Weintraub, S., Guinessey, J. E., \& Mesulam, M. M. (1992). Diminished curiosity in patients with probable Alzheimer's disease as measured by exploratory eye movements. Neurology, 42(2), 320-320.

Engel, S. (2011). Children's need to know: Curiosity in schools. Harvard Educational Review, 81(4), 625-645.

Forestier, S., Mollard, Y., \& Oudeyer, P. Y. (2017). Intrinsically motivated goal exploration processes with automatic curriculum learning. arXiv preprint arXiv: 1708.02190.
Gilbert, S. J. (2015). Strategic use of reminders: Influence of both domain-general and task-specific metacognitive confidence, independent of objective memory ability. Consciousness and Cognition, 33, 245-260.

Gordon, G., Breazeal, C., \& Engel, S. (2015, March). Can children catch curiosity from a social robot?. In 2015 10th ACM/IEEE International Conference on Human-Robot Interaction (HRI) (pp. 91-98). IEEE.

Gruber, M. J., Gelman, B. D., \& Ranganath, C. (2014). States of curiosity modulate hippocampus-dependent learning via the dopaminergic circuit. Neuron, 84(2), 486-496.

Haith, M. M. (1980). Rules that babies look by: The organization of newborn visual activity. Lawrence Erlbaum Associates, New Jersey.

Henderson, B., \& Moore, S. G. (1980). Children's responses to objects differing in novelty in relation to level of curiosity and adult behavior. Child Development, 51(2), 457-465.

Hunter, M. A., \& Ames, E. W. (1988). A multifactor model of infant preferences for novel and familiar stimuli. Advances in infancy research.

Kang, M. J., Hsu, M., Krajbich, I. M., Loewenstein, G., McClure, S. M., Wang, J. T. Y., \& Camerer, C. F. (2009). The wick in the candle of learning: Epistemic curiosity activates reward circuitry and enhances memory. Psychological Science, 20(8), 963-973.

Kornell, N. (2009). Metacognition in humans and animals. Current Directions in Psychological Science, 18(1), 11-15.

Kornell, N. (2014). Where is the meta in animal metacognition? Journal of Comparative Psychology, 128(2), 143-149.

Kruger, J., \& Dunning, D. (1999). Unskilled and unaware of it: How difficulties in recognizing one's own incompetence lead to inflated self-assessments. Journal of Personality and Social Psychology, 77(6), 1121-1134.

Loewenstein, G. (1994). The psychology of curiosity: A review and reinterpretation. Psychological Bulletin, 116(1), 75-98.

Marti, L., Mollica, F., Piantadosi, S.T., \& Kidd, C. (2019) Certainty is primarily determined by past performance during concept learning. Open Mind.

McDonnell, J.V., Martin, J.B., Markant, D.B., Coenen, A., Rich, A.S., and Gureckis, T.M. (2012). psiTurk (Version 1.02) [Software]. New York, NY: New York University. Available from https:/github.com/ NYUCCL/psiTurk

McPhetres, J. (2019). Oh, the things you don't know: awe promotes awareness of knowledge gaps and science interest. Cognition and Emotion, 1-17.

Montessori, M. (1964). The Montessori Method, Rome 1912. Oxford, England: Bentley, Inc..

Nelson, T. O., \& Narens, L. (1990). Metamemory: A theoretical framework and new findings. In G. Bower (Ed.), The psychology of learning and motivation: Advances in research and theory (Vol. 26, pp. 125-173). New York: Academic Press.

Oudeyer, P. Y. (2004). Intelligent adaptive curiosity: A source of self-development. In Berthouze, L., Kozima, H., Prince, C. G., Sandini, G., Stojanov, G., Metta, G., and Balkenius, C. (Eds.) Proceedings of the Fourth International Workshop on Epigenetic Robotics Lund University Cognitive Studies, 117, ISBN 91-974741-3-4.

Oudeyer, P. Y., \& Kaplan, F. (2006). Discovering communication. Connection Science, 18(2), 189-206.

Reio Jr, T. G., \& Wiswell, A. (2000). Field investigation of the relationship among adult curiosity, workplace learning, and job performance. Human Resource Development Quarterly, 11(1), 5-30.

Reio, T. G. (2004). Prior knowledge, self-directed learning readiness, and curiosity: Antecedents to classroom learning performance. International Journal of Self-Directed learning, 1(1), 18-25.

Roder, B. J., Bushnell, E. W., \& Sasseville, A. M. (2000). Infants' preferences for familiarity and novelty during the course of visual processing. Infancy, 1(4), 491-507. 
Schulz, L. E., \& Bonawitz, E. B. (2007). Serious fun: Preschoolers engage in more exploratory play when evidence is confounded. Developmental Psychology, 43(4), 1045-1050.

Schwartz, B. L., \& Metcalfe, J. (1992). Cue familiarity but not target retrievability enhances feeling-of-knowing judgments. Journal of Experimental Psychology: Learning, Memory, and Cognition, 18, 1074-1083.

Sobel, D. M., Yoachim, C. M., Gopnik, A., Meltzoff, A. N., \& Blumenthal, E. J. (2007). The blicket within: Preschoolers' inferences about insides and causes. Journal of Cognition and Development, 8(2), 159-182.
Stahl, Aimee E., and Lisa Feigenson. "Observing the unexpected enhances infants' learning and exploration." Science 348.6230 (2015): 91-94.

Wilcox, R. R. (2009). Comparing Pearson correlations: Dealing with heteroscedasticity and nonnormality. Communications in Statistics: Simulation and Computation, 38(10), 2220-2234.

Publisher's note Springer Nature remains neutral with regard to jurisdictional claims in published maps and institutional affiliations. 